\title{
Arbor
}

\section{La organización de grandes eventos deportivos}

\section{Vicente Añó Sanz}

Arbor CLXV, 650 (Febrero 2000), 265-287 pp.

El deporte moderno ha evolucionado sustancialmente en los últimos años, principalmente en los aspectos económicos y organizativos. La clave para ello ha sido la gran demanda de los ciudadanos hacia el mismo. Ello ha provocado su comercialización y la organización de grandes acontecimientos deportivos, que como los JJOO o los Campeonatos del Mundo, obligan a crear modelos de organización en los que se distribuyen las áreas de trabajo para resolver las tareas que se plantean, como los transportes y alojamientos de la gran cantidad de personas que acuden, los aspectos técnicos deportivos, los medios materiales, los recursos humanos, etc.

\section{Introducción. ¿Qué es un gran evento?}

Las actividades o los acontecimientos de todo tipo que se organizan en torno al deporte son muy diversos y tienen necesidades muy distintas, de manera que es muy difícil englobar todos ellos bajo el mismo epígrafe. En principio cabe distinguir entre lo que es una práctica del deporte como recreación, salud, o educación de lo que es la práctica competitiva, aunque esta sea de carácter popular. En estas cabe hablar de acontecimientos deportivos en algunos casos, pero no siempre.

Por ello, en esta introducción vamos a tratar de delimitar que es un gran evento deportivo, sin entrar en la dicotomía internacional/na- 
cional, dado que en ambos casos podemos encontrarnos con una actividad de gran relieve.

\subsection{La evolución de la organización deportiva}

El Deporte ha experimentado un cambio espectacular en su organización y, sobretodo, en su sistema de financiación y ha pasado del «amateurismo» del clásico mecenas que otorgaba una subvención para desarrollar una actividad deportiva a vender por miles de millones de pesetas los derechos de retransmisión de sus competiciones, el derecho de uso de su propia imagen (logo, mascota, etc.), y, por supuesto, a cobrar la asistencia a algunos deportes con cantidades elevadas. Este cambio tan significativo ha provocado, a su vez, otros sustanciales en la organización del deporte en general y en la organización de acontecimientos deportivos.

Sin embargo, este cambio no se ha operado lentamente como ha ocurrido con otras actividades sociales, económicas o políticas, sino, a mi entender, bruscamente, en los últimos 15 años: los que van desde la elección de Samaranch y la celebración de la olimpíada de Los Angeles ${ }^{1}$ hasta nuestros días y, en ese sentido, el deporte como organización tendría una historia reciente.

Sería una historia, además, sin excesiva complejidad organizativa hasta esos últimos años señalados, al contrario de lo que ha ido sucediendo en las organizaciones sociales modernas, tal y como señalan autores como Mintzberg (1983) o Peiró (1986) . La primera olimpiada con una organización más compleja sería la de 1936 en Berlín, que se adelantaría varios lustros a su época por motivos que podríamos llamar de «escaparate político» y de utilización del deporte para propagar determinadas ideologías (Mandell, 1986; Brohm, 1976; Elias y Dunning, 1992).

Pero, en estos inicios, el deporte llevaba ya consigo un enorme caudal de competitividad y producción, que hoy metemos de lleno dentro del sector servicios - culturales y deportivos- y, desde el principio, amenazó con convertirse en el acontecimiento social de mayor nivel de audiencia e impacto del siglo XX.

No obstante, al filo de 1980, nadie quería organizar unos JJOO. Los elevados gastos de los precedentes, México 68, Munich 72 y, sobretodo, Montreal 76, unido a las complicaciones políticas y sociales de las tres ${ }^{2}$ hizo que Moscú-80 y Los Ángeles-84 fueran candidaturas únicas. 
Desde la elección de Samaranch, las perspectivas olímpicas comenzaron a cambiar. Todavía en 1984 Los Angeles fue boicoteado en respuesta al que planteó anteriormente Estados Unidos y países aliados a Moscú-80, pero ahí comenzó un espectacular cambio de tendencia: fue la primera olimpíada de la historia que obtuvo beneficio económico y que fue organizada de forma casi totalmente privada.

Los cambios comenzaron a experimentarse antes, posiblemente el primer indicio se experimento en la olimpiada de México, de gran impacto mundial gracias al alcance de las retransmisiones televisivas, la gran mejora de los medios técnicos y la espectacularidad de los records conseguidos por los deportistas, lo que permitió catapultar el deporte a escala mundial.

Desde entonces la organización del deporte se hizo cada vez más compleja y acaba convirtiéndose en UNA ORGANIZACIÓN SOCIAL PRODUCTIVA, merced a dos condiciones:

a) al proceso de actualización de las «rígidas» normas de participación olímpica amateur.

b) al creciente interés social por el espectáculo deportivo.

En la primera tuvo una eficaz contribución J. A. Samaranch, quien tuvo la agilidad necesaria para adaptar el movimiento olímpico a los nuevos tiempos. Sus detractores le acusan de comercializar el mundo del deporte (Simson y Jennings, 1992), pero ciertamente de no haberlo hecho posiblemente se habría desmembrado y no tendría el alcance actual. Otros autores, como Mullin, Hardy y Sutton (1995) reconocen que el cambio en las normativas vigentes permitió a la olimpíada de Los Angeles tener beneficios.

En cuanto al segundo aspecto es obvio la evolución del interés por el espectáculo deportivo, de manera que hoy no existe ningún otro que se le pueda comparar ni en asistencia directa de público ni en el nivel de telespectadores.

\subsection{El deporte como el mayor acontecimiento mundial de finales del siglo $X X$}

Definir el deporte como el mayor acontecimiento social de final de siglo no es baladí, sino algo que se demuestra continuamente con la asistencia directa a los recintos deportivos y con la enorme cantidad de personas que lo ven por la Televisión.

De este modo, podemos comprobar como la Olimpíada de Barcelona fue seguida por 16.600 millones de telespectadores de audiencia acu- 
mulada durante sus 16 días de duración a una media de 1.037 millones de personas diarias. El campeonato del Mundo de Fútbol de 1990 fue seguido por 26.693 millones de personas (513 millones por día), mientras que en lo que al atletismo se refiere es el tercer acontecimiento mundial más seguido, de manera que acumuló en el campeonato del mundo de Goteborg (1995) 3.681 millones, a una media de 460.1 personas por día.

Los años 90 han supuesto un salto cualitativo esencial en estas audiencias que han ido creciendo a pesar de la magnitud de las mismas, de manera que la olimpiada de Atlanta tuvo valores similares a la de Barcelona, pero con un total de 20.000 millones de telespectadores de audiencia acumulada. El mundial de Fútbol de Francia de 1998 obtuvo alrededor de 40.000 millones de telespectadores. Y el mundial de Atletismo de Sevilla de 1999 rebasó la barrera de los 4.000 millones de telespectadores de audiencia acumulada. En algunos países como Gran Bretaña obtuvo una punta de 8,6 millones de audiencia, el segundo nivel más alto de 1999. En Alemania, la ZDF, que poseía los derechos de retransmisión logró 6,5 millones de telespectadores durante la prueba de Triple salto (el campeón fue de este país), superando al fútbol que sólo alcanzó 4,5 millones.

En España, el seguimiento deportivo en general también es impresionante, pero está muy escorado hacia el mundo del fútbol, muy por encima de lo que ocurre en otros países,. aunque, no obstante el Mundial de Atletismo de Sevilla ha alcanzado un total de 27.5 millones de telespectadores sólo en TVE, a pesar de coincidir con las dos primeras jornadas de la Liga de fútbol, colocándose por encima de los partidos televisados en ambas jornadas.

De este modo, los partidos del Real Madrid- Barcelona alcanzaron una cifra en la temporada 98/99 de 8.252.000. La final europea entre el Real Madrid y la Juventus obtuvo 10.737 .000 telespectadores (en la repetición posterior llegó a alcanzar los 13.229 .000 telespectadores con un porcentaje medio del $35.5 \%$, siendo el programa más visto de 1998).

La selección española tuvo su máxima audiencia durante el Mundial de Francia de 1998 en el partido contra Nigeria que alcanzo los 12.002.000 telespestadores con una cuota de pantalla del $31.3 \%$. De máxima audiencia fue, también, el Barcelona- Mallorca de la final de la Copa que logró 11.005 .000 telespectadores. En total la retransmisión de 6 partidos de fútbol durante 1998 fueron los programas más visto en Tv, por encima de Médico de Familia (8.996.000 telespectadores en su program? más visto). 


\section{La organización de grandes eventos deportivos}

El resto de los deportes queda muy lejos de estas cifras, y ningún deporte se colocó entre los 50 programas más vistos en 1998. Sólo el Tour, en la época de Indurain se le podía acercar. El de 1995 arrojó una cifras de 4.447.300 Telespectadores de media, con una cuota de pantalla del $47.3 \%$, alcanzado en los días más importantes una cifra de 6.175.000 personas y una cuota de pantalla de $61.1 \%$.

El atletismo lo consigue esporádicamente como en el Mundial de Sevilla del verano de 1999, que alcanzó cotas excepcionales: 27.559.000 telespectadores de audiencia acumulada, 5.289 .000 durante el marathon con una cuota de pantalla del $65,2 \%$, obteniendo la prueba de 1500 una audiencia media de 3.541 .000 telespectadores con un $44,3 \%$ de cuota de pantalla.

En cuanto a otros medios de comunicación, los programas de radio de mayor audiencia alcanzan cotas entre el millón y el millón y medio de radioyentes. Y la prensa tiene como lider al diario Marca con más de 2 millones de lectores diarios (en 1997 vendió 495.915 ejemplares diarios, el mayor del país), el As supera el medio millón (107.169 ejemplares diarios vendidos en 1997) y el Sport de Barcelona se acerca a esta cifra (105.537 de venta diaria).

Todas estas cifras nos indican la importancia que tiene en los albores del siglo XXI el deporte, y dentro del mismo, los agrandes acontecimientos deportivos, retransmitidos al universo entero gracias a la TV.

\subsection{Condiciones que debe reunir un gran acontecimiento}

Los acontecimientos deportivos son muy diversos y no podemos conceptuar como tales todas las actividades deportivas, de manera que podemos clasificar las actividades deportivas principalmente en tres grandes grupos desde el punto de vista competitivo:

a) ACTOS PUNTUALES

b) GRUPOS DEPORTIVOS

c) ACTOS PERMANENTES

Como su propio nombre indica los actos puntuales son aquellos que se organizan esporádicamente, una vez al año, aunque se repitan todos los años y siempre en las mismas fechas. Son actividades que no tienen continuidad muchos días o semanas. Es, posiblemente, el grupo con mayor número de actividades, puesto que, salvo las ligas de clubes, el resto de actividades pueden tener este calificativo. Son los marathones, las carreras urbanas, los Grandes Premios de ciclismo, motociclismo, atletismo e, incluso, el tenis o las vueltas ciclistas. 
Por su parte, la actividades de los grupos deportivos podrán ser de carácter permanente o puntual como las que organizan con carácter esporádico grupos de montaña o de cicloturismo, etc. Las de caráácter permanente son las más habituales en los grupos deportivos y tienen una doble vía: externa e interna. En el primer caso se trata de los partidos que celebran cada semana o incluso entre semana, pero con una periodicidad continuada a lo largo del año, y, en el segundo caso, son actividades cotidianas de entrenamiento o de servicio hacia los miembros del club, del equipo, o del gimnasio, en su caso.

Esta última actividad de los grupos deportivos es lo que se puede conceptuar como acto permanente y aunque reúne aspectos del primer tipo de actividad, puesto que organiza un acto puntual un día a la semana, al mes o cada quince días, éste se repite de modo constante. Es el caso de las ligas de los deportes de equipo. Vendría a ser su actividad externa.

No obstante, para que una actividad deportiva, pertenezca al grupo que pertenezca, pueda ser considerada como acontecimiento debe reunir una serie de condiciones entre las que podemos citar las siguientes:

1) repercusión social

2) nivel de asistencia de público

3) presencia en los medios de comunicación

4) nivel de audiencia televisiva

A estos cuatro factores habría que añadir otros más específicos de un deporte concreto que contribuyen a completar si una actividad deportiva permite ser clasificada como acontecimiento. Estos serían:

5) tipo de deporte

6) dificultad de la práctica

El primero condiciona ya la calificación como posible acontecimiento o no en función de su nivel de espectacularidad o la capacidad para realizarse públicamente, de manera que se sabe que el fútbol o el baloncesto arrastra de entrada determinados niveles de audiencia, mientras que las actividades subacuáticas o el montañismo, por poner casos extremos, difícilmente se realizan cara a un público, siquiera mínimo.

Estas dos mismas actividades reseñadas nos vuelven a servir de ejemplo para analizar el siguiente factor: el de la dificultad de la práctica. Cuanto más difícil sea un deporte más dificultades de contar con una masa de practicantes tendrá y, por tanto, de seguidores. Y si es un gran acontecimiento escalar el Everest, los es en tanto que aventura deportiva y no como acontecimiento o espectáculo deportivo. 


\section{La organización de grandes eventos deportivos}

Todos ellos nos llevarán finalmente a otros dos elementos fundamentales que acabarían por dar realce a la actividad y que serían el exponente máximo por el que una actividad deportiva pasa no sólo a ser un acontecimiento sino un gran acontecimiento deportivo:

7) patrocinadores

8) ingresos propios (taquillas, venta de objetos, etc.)

Estos dos últimos factores como he indicado son una consecuencia de los anteriores, pero vienen a demostrar el alcance del acontecimiento. Todos los grandes acontecimiento deportivos cuentan en la actualidad con importantes compañías comerciales que les patrocinan y en muchas de ellas se obtienen buenos ingresos por taquilla o por la venta de objetos de recuerdo conmemorativos (merchandising).

Un buen ejemplo de ello es los más de 1000 millones de pesetas que obtuvo la Selección Española de Fútbol con el patrocinio del equipo para el último Campeonato del Mundo de Fútbol de Francia en 1998 o las importantes empresas que patrocinan la propia competición.

Por todo ello, podemos definir un gran evento o acontecimiento deportivo como «aquella actividad deportiva que cuenta con un alto nivel de repercusión social traducido en una fuerte presencia en los medios de comunicación y que genera por si misma ingresos económicos».

Esta definición genérica permite englobar múltiples acontecimientos o eventos deportivos, ya que no especificamos en la misma ni el alcance de la presencia de los medios de comunicación, ni el de los ingresos económicos, ni el concepto.

\subsection{Tipos de acontecimientos}

Entrar en una clasificación de acontecimientos o eventos deportivos es una tarea ardua porque existen muchos deportes y dentro de ellos ctividades muy diversificadas.

De este modo, lo primero que podemos hacer es tratar de plasmar tipos de espectáculos que existen en el mundo del deporte para, posterioridad, tratar de agruparlos en grupos más amplios.

Analizando los diferentes deportes podemos encontrar 13 tipos de espectáculos diferentes, tal y como vemos en Cuadro 1.

Esta clasificación de los eventos deportivos que existen en la mayoría de los deportes va de lo sencillo a lo complicado en cuanto a organización $\mathrm{y}$, en cierto modo, también en los niveles de audiencia y repercusión de un evento, así como en los costes o ingresos que genera. No obstante, hay bastantes excepciones, sobretodo en los acontecimiento permanentes 
de las ligas de los equipos, pues un partido del Barcelona y el Madrid, aunque sea una actividad de carácter habitual, es siempre un acontecimiento que generará un nivel de atención y de ingresos muy superior a otros partidos, incluso de esos mismos equipos.

Cuadro 1. Tipos de espectáculos

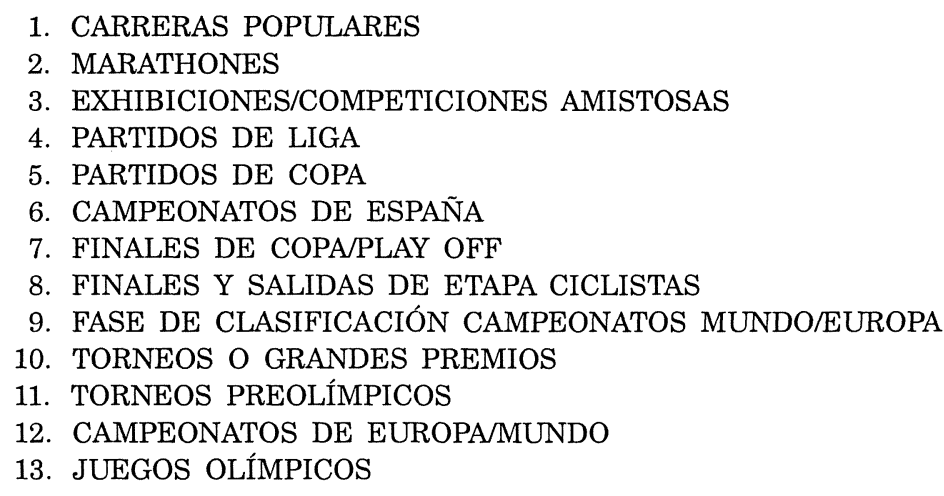

Pero, evidentemente, el nivel de complejidad organizativa de un partido de liga es mucho menor que el de una carrera popular en muchas ocasiones. Lo que ocurre es que el nivel de audiencia y asistencia en directo permite clasificarlos en un segundo grupo más complejo.

De acuerdo, pues, con esta clasificación las carreras populares y las marathones serían los primeros eventos que nos podemos encontrar en el mundo del deporte, debiendo precisarse que no todas ellas los serán y que hace unos pocos años casi ninguna podía calificarse de esta manera. Pero ese mundo ha cambiado mucho y algunas carreras reúnen importantes masas de participantes (la Volta a Peu a Valencia alcanza los 50.000, la Cursa de Barcelona pasa de los 100.000) que implica un elevado coste económico su organización y que tiene una buena presencia en los medios de comunicación.

Por otra parte, organizar un campeonato de España de cualquier deporte resulta más complicado que los partidos de liga, algunos tienen un alcance puntual superior, pero en general generan menos recursos y menor audiencia, que los deportes más conocidos.

La clasificación o diferenciación de los acontecimientos deportivos de entre los apartados 6,7, 8, 9 y 10 es difícil de realizar porque son competiciones muy diferentes entre sí y dentro de ellas mismas, pero la diferencia entre finales de Copa/Play Off, Salidas y Llegadas de 


\section{La organización de grandes eventos deportivos}

vueltas Ciclistas o los Torneos Preolímpico o un Gran Premio de motociclismo o tenis, se puede realizar en base a su nivel de importancia deportiva. Los primeros son de tipo nacional 6 y 7) y los segundos (8, 9 y 10) son de carácter internacional.

Así las cosas, todos estos tipos de acontecimientos nos permiten agruparlos en 4 grupos de espectáculos:

a) puntuales

b) habituales

c) puntuales extraordinarios

d) puntuales de gran impacto

En el primer grupo nos encontraríamos con espectáculos de tipo mínimo o de un nivel de complejidad 1 o menor, tales como carreras populares, maratones, meetines de atletismo y algún que otro Gran Premio, es decir los grupos 1, 2, 8 y 10. Al segundo grupo pertenecen los partidos habituales de las ligas de deportes colectivos, principalmente, y otros de características similares que hemos encuadrado en los tipos de espectáculos $3,4,5,6$ y 7 . Su nivel de complejidad es 1 ó 2, menor o medio, en función del partido concreto de que se trate que llevará muchos o pocos espectadores al campo, se televisará o no, produciendo una gran diferencia dentro del mismo grupo entre unas $\mathrm{u}$ otras actividades.

En cuanto a los grupos 3 y 4, puntuales extraordinarios y puntuales de gran impacto, el número de espectáculos o competiciones a encuadrar es mucho menor. En el primer caso nos encontramos con las fases clasificatorias de campeonatos de Europa o del Mundo, con Torneos Preolímpicos y algunos campeonatos del mundo y su nivel de complejidad puede ser 2 ó 3, medio o máximo. En el último grupo, de máxima nivel de complejidad o nivel 3, se encuadran los Juegos Olímpicos o los Campeonatos del Mundo de Fútbol, casi exclusivamente.

\section{La organización de un gran evento}

Una vez analizadas las características del deporte actual y las condiciones que debe reunir un gran evento, así como su tipología, entramos en este apartado en la organización, propiamente dicha, de un gran acontecimiento o evento deportivo, desde lo que supone la candidatura previa y la estructura organizativa, a la ejecución final. 


\subsection{Candidatura y Campaña previa}

La organización de un gran evento deportivo comienza con la elaboración de la candidatura y la campaña previa a la concesión del evento. Es la primera fase de la ejecución, incluso, del propio evento por dos razones. En primer lugar, porque en muchos casos no se pasará de ahí y los objetivos deben plantearse si ese caso se produce y, en segundo lugar, porque las acciones que se desarrollen durante la presentación de la candidatura, las ofertas que se hagan, los apoyos que se obtengan servirán para el desarrollo de la organización posteriormente.

Así pues, la preparación de la candidatura y la campaña pública en torno a la misma es muy importante para conseguir el evento y para su posterior desarrollo.

Esta fase puede dividirse en de 5 pasos, que serían los siguientes:

1. Trabajo oculto

2. Lanzamiento del proyecto

3. Campaña de difusión nacional

4. Campaña de difusión internacional

5. Organización de actos.

El primer paso es más importante de lo que parece y es el que condicionará el éxito o el fracaso de las posteriores campañas. Es un trabajo que no tiene porqué trascender públicamente y que se dirige a conseguir el apoyo institucional, tanto público como privado. Se trata primeramente de poner de acuerdo a los distintos organismos que pueden colaborar o que tienen competencias en el tema.

En esta fase se trata de plantearse un aspecto fundamental de la candidatura: la filosofía de la misma. Es decir: porqué y para qué se pide un gran acontecimiento, cuales son las posibilidades de obtenerlo, las líneas básicas para lograrlo y quienes están en situación de otorgarlo. Y, por supuesto, tener diseñadas las primeras actuaciones tanto para el caso de la concesión como para su denegación.

En este aspecto, por ejemplo, la candidatura de Sevilla a la olimpiada del 2004 fue sustancialmente distinta a la que se plantea la misma Ciudad para el 2008. En el primer caso existían posibilidades de obtener la nominación ${ }^{3}$ ya que todos los pronósticos apuntaban a que una ciudad europea sería la ganadora, como así fue (Atenas). Para el 2008 los pronósticos previos y la historia olímpica indican que ninguna ciudad europea obtendrá la nominación, que se conocerá a finales del $2001^{4}$. 


\section{La organización de grandes eventos deportivos}

A continuación, resuelto este primer aspecto filosófico y programático se debe pasar al lanzamiento público del proyecto, teniendo en cuenta la elección del momento para hacerlo.

El lanzamiento público del proyecto debe venir acompañado de una mínima estructura organizativa, de dotación presupuestaria, de un calendario de actividades y de un local con su infraestructura correspondiente.

De forma inmediata el lanzamiento público debe acompañarse de un calendario de actividades en los que se lleve a cabo la presentación de la candidatura a nivel local, nacional e internacional, pues debemos tener en cuenta que un aspecto sustancial de la candidatura, que permitirá saber su grado de afianzamiento, será el convencimiento, la asunción y el apoyo de los propios ciudadanos de la sede de la candidatura. Si ellos no están convencidos o no conocen el evento, más difícil será darlo a conocer a otros o que la apoyen.

El último paso de la campaña previa de la candidatura es la organización de actos o actividades. Aunque viene en último lugar, ello no implica que empiece mucho después de la presentación de la candidatura sino que debe hacerse de forma inmediata.

Los actos susceptibles de organizarse son de características muy diversas, pero básicamente pueden ser de tres tipos:

a) actos protocolarios de presentación propia

b) actos protocolarios que acompañan otros eventos

c) organización o patrocinio de competiciones

Los primeros son actos propios, específicos, que monta el Comité de Campaña sin que exista ningún tipo de acontecimiento deportivo o de reunión federativa, olímpica o congresual, a diferencia del segundo tipo que son actos de presentación que realizan aprovechando la celebración de algún campeonato, sea este nacional o internacional.

Por último, la organización o patrocinio de competiciones es una forma muy interesante de difundir una candidatura y han dado buenos resultados. En eventos internacionales del tipo de una olimpiada, por ejemplo, la organización o el patrocinio de campeonatos de algunos deportes ayuda al conocimiento internacional y sirve de base curricular a la candidatura. Por ejemplo, Barcelona 92 pidió, antes de su concesión, la organización de la Copa del Mundo de Atletismo para 1989, con la que se inauguró el Estadio Olímpico antes de su concesión y Sevilla 2004 apoyó la petición hispalense de celebrar el Campeonato del Mundo de Atletismo para 1999, que además finalmente fue una magnífica compensación y realidad a la no concesión de la olimpiada. 


\subsection{Estructura organizativa}

Una vez concedida una candidatura el primer trabajo es la creación del Comité Organizador y el montaje de una estructura organizativa con todas las necesidades de infraestructura que conlleva (locales, material burocrático, material de papelería y fungible, dotación de aparatos informáticos, etc.).

En la mayoría de los casos, parte de los miembros del Comité de Campaña continúan y se hacen cargo de las diferentes áreas, se amplían éstas y se contrata o se pide la colaboración de otras personas. Es conveniente, en este sentido, tener parte de la infraestructura ya montada previamente o, al menos, el diseño de la misma, lo cual, además será una exigencia del organismo que concede la competición.

Ello debe ser así, porque en la mayoría de las grandes competiciones no existe tanto tiempo de preparación como en una olimpiada y se conceden, a veces, dentro del mismo año de organización - eventos nacionales- o con uno o dos años previos a la celebración.

En ese sentido, por ejemplo, uno de los mayores problemas que tuvo el Campeonato del Mundo de Atletismo Sevilla-99 fue el escaso tiempo de preparación del mismo desde la concesión (marzo del 97) a la celebración (agosto del 99) para un acontecimiento de esa envergadura ${ }^{5}$. Y todo ello sin estadio en el momento de la adjudicación, el cual fue construido en esos 2 años.

La estructura organizativa puede ser muy distinta en el caso de que el evento se organice desde un Comité preparado al efecto, o desde empresas u entidades subsidiarias del evento.

En esta línea, podemos podemos encontrarnos con 4 tipos de estructuras de organización, que son las siguientes:

1. GESTIÓN DIRECTA

2. EMPRESAS ADJUDICATARIAS

3. CLUBS O GIMNASIOS

4. GESTIÓN MIXTA

En el primer caso, el organismo u organismos a quienes se concede el acontecimiento lo organizan directamente montando un Comité específico o una empresa para llevar a cabo el mismo (caso de Barcelona 92, Atlanta 96,Sevilla 99...). En el segundo caso, el acontecimiento es cedido a una empresa de gestión que se encarga de toda la organización. Es el caso de algunos acontecimientos muy significativos como la Vuelta Ciclista a España o el Campeonato del Mundo de Motociclismo, donde 


\section{La organización de grandes eventos deportivos}

empresas originariamente de publicidad (Unipublic y Dorna) se encargan de todo el montaje.

El tercer caso es típico de las competiciones de liga o cuando se celebra un competición de deportes colectivos con varias subsedes en el caso de los clubes (lo que pasó con el fútbol de Barcelona 92), y de las competiciones de judo, karate, gimnasia rítmica y deportes similares en el caso de los gimnasios.

La gestión mixta queda para los casos en los que se monta un Comité Organizador, pero cede algunas parcelas a empresas privadas o instituciones al margen. Suele ser el caso de la captación de publicidad del evento o del merchandising, que se suele adjudicar a empresas de publicidad o de gestión. $\mathrm{O}$ el caso, por ejemplo, del Campeonato del Mundo de Atletismo en pista cubierta de Sevilla 91, donde la Expo.92 se encargó de toda el área de prensa.

En el caso de comités de organización propios lo habitual en las grandes competiciones es un tipo de estructura jerarquizada con un Director General o Gerente en la cúpula y dos o cuatro directores de grandes áreas, debajo de los cuales se distribuyen parcelas más específicas de trabajo.

En competiciones de tamaño medio, no es necesaria una estructura tan jerarquizada y piramidal, sino que lo habitual es nombrar un coordinador o director general y debajo de él tantas áreas de trabajo como se considere oportunas. Este tipo de estructura es más rápido y sencillo y óptimo para este tipo de competiciones no demasiado complejas.

\section{3. Áreas de trabajo}

Las áreas de trabajo de un evento de cierta magnitud deben contemplar todos los aspectos que rodean al mismo, desde los publicitarios a las posibles publicaciones que haya que hacer, el control de los gastos, los medios de transporte, los alojamientos, etc.

Igualmente se hace necesario que, independientemente del tipo de estructura formal que se establezca, existan responsables al frente de cada una de ellas y tantas subáreas y personal humano como sea necesario.

De este modo, las áreas concretas en que podría dividirse el trabajo de un Comité Organizador podemos contemplarlas en el Cuadro 2 
CUADRO 2. Áreas de organización

\begin{tabular}{|l|l|}
\hline \multicolumn{1}{|c|}{ ÁREAS } & \multicolumn{1}{c|}{ ÁREAS } \\
\hline 1. MARKETING & 9. INSTALACIONES \\
2. ECONÓMICA Y ADMINISTRACIÓN & 10. TÉCNICA \\
3. RECURSOS HUMANOS & 11. SEGURIDAD \\
4. CEREMONIAS & 12. MÉDICA \\
5. PROMOCIÓN & 13. ALOJAMIENTOS \\
6. MEDIOS COMUNICACIÓN & 14. TRANSPORTES \\
7. PUBLICACIONES & 15. ACREDITACIONES \\
8. PROTOCOLO & \\
\hline
\end{tabular}

En la parte izquierda de la tabla nos encontramos con las áreas propiamente económicas y de proyección pública del acontecimiento, mientras que en la parte derecha se encuentran las de infraestructura y de tipo técnico, como una posible gran subdivisión aunque en realidad pueden ser cuatro el agrupamiento de estas macroáreas, que podrían ser:

a) área económica (marketing, economía, administración y recursos humanos).

b) área de promoción y divulgación (promoción, medios de comunicación, publicaciones y protocolo)

c) área técnica y de instalaciones (técnica-deportiva, instalaciones: arquitectura/ingeniería)

d) área de servicios (alojamientos y transportes, servicios médicos, seguridad, servicios tecnológicos).

Un caso real muy reciente ha sido la agrupación en macroáreas, llamadas divisiones, llevado a cabo por la organización del Campeonato del Mundo de atletismo en Sevilla 99, similar al anterior, pero con sus diferencias específicas:

a) Logística (voluntarios, servicios médicos, acreditaciones).

b) Alojamientos y Transportes (alojamientos y transportes)

c) Medios de Comunicación (medios de comunicación, publicaciones y ceremonias).

d) Área técnica- deportiva (horarios, pruebas, jueces, resultados.....)

e) Marketing (patrocinadores, merchandising, calle del ocio)

f) Régimen interior (obras menores, aprovisionamientos, seguridad, informática).

En la estructura de trabajo de Sevilla 99 estas divisiones dependían de un Coordinador General, que contaba con un Adjunto del cual dependía otra macroárea que englobaba la promoción, el gabinete de 


\section{La organización de grandes eventos deportivos}

prensa y las actividades culturales e inicialmente el protocolo, que luego se desgajó para tener una autonomía propia. Finalmente, el área de economía y administración dependía directamente del Coordinador General que contaba con un responsable de esta área. La coordinación de las áreas de trabajo que se realizaba a través de un jefe de gabinete.

Esta distribución del sistema de trabajo se integró en un órgano de funcionamiento colegiado que se denominó Comité Permanente y que se reunía una vez a la semana en pleno, pero ocupaba la misma dependencia y su relación era diaria. A su vez, este Comité Permanente dependía del Comité Organizador, formado por los representantes políticos e institucionales.

Individualmente, todas estas áreas de trabajo pueden tener diversas alternativas de integración u organización. Por ejemplo, el área de marketing podría agruparse junto a la de Promoción, pero en muchos eventos permanece separada porque una se encarga más de las cuestiones de imagen del evento, de la explotación de los derechos del mismo y de atender los planteamientos, exigencias y las concesiones que se realizan a los patrocinadores y la otra de la difusión concreta del acontecimiento en la propia Ciudad donde se realiza, principalmente (banderolas, carteles, pancartas, publicidad en los autobuses, etc.).

Ambas áreas, no obstante, trabajan en estrecha colaboración entre sí y con el área económica, responsable de la elaboración de los presupuestos, la captación de recursos y el control y disponibilidad del gasto, para lo que cuenta con el apoyo del área de administración y contabilidad.

El área de medios de comunicación también tiene connotaciones con la de promoción, pero tiene suficiente entidad por si misma. De hecho, en Sevilla 99 estuvieron inicialmente juntas ya que dos son los grandes campos de trabajo de esta área:

a) la infraestructura necesaria para los medios de comunicación (sala de prensa, tribuna de prensa-radio-Tv en la zona del evento, zona para las Unidades Móviles (Compound), recursos tecnológicos, etc.).

b) la atención propia a los medios de comunicación y la información a los mismos sobre todos los acontecimientos que se vayan produciendo en torno a la organización

Por otra parte, un área de suma importancia en cualquier evento deportivo es la de recursos humanos, la mayor parte de ellos voluntarios. 
Los recursos humanos de los acontecimientos deportivos pueden ser de 5 tipos diferentes:

1.-EJECUTIVOS

2.-PERSONAL DE SERVICIO

3.-PERSONAL TÉCNICO

4.-VOLUNTARIOS

5.-SEGURIDAD

El primero de ellos es el menos numeroso, pero sobre el que recae las decisiones estratégicas que determinarán el éxito o el fracaso del acontecimiento. Son fundamentalmente el Director General o Gerente, los directores de área, de división, etc.

El personal de servicio, gran parte del mismo contratado de forma permanente o eventual, son los que realizan las tareas más ingratas, posiblemente de los acontecimientos. Son camareros/as, limpiadores/as, porteros/as, aposentadores/as, taquilleros/as, electricistas, etc. En algunos casos estos puestos pueden ser ocupados por voluntarios, pero no es lo habitual, salvo, quizás, el de aposentadores que sí lo suelen hacer.

En cuanto al personal técnico suele ser de cuatro tipos, frecuentemente:

a) deportivos que elaboran los calendarios, normas de competición, etc., y que suelen ser profesionales del deporte (licenciados en Ciencias de la A. Física y el Deportede E. Física), entrenadores e, incluso, árbitros o jueces.

b) árbitros o jueces que velan por el cumplimiento de los reglamentos,

c) especialistas (médicos, informáticos, ingenieros, arquitéctos...)

El grupo más numeroso y el más imprescindible, a la postre, son los voluntarios que colaboran con la organización de un evento deportivo. Suelen realizar las tareas más diversas: desde acompañar a los equipos durante su estancia en la Ciudad sede de la competición, hacer de choferes, ayudantes de los árbitros o jueces, cuidadores de las instalaciones o de la ropa de los participantes, y sobretodo son el soporte básico de casi todos las áreas, pues se encargan de acreditar a los participantes, de atender a la prensa, a los vips, etc., etc. En Sevilla 99 se hizo una campaña de captación de voluntarios con una respuesta esplendida ya que capto a 6.486 personas, de las que, finalmente, intervinieron 3.230 .

Por último, el personal de seguridad varía mucho de unas competiciones a otras. No es lo mismo celebrar unos juegos olímpicos o un campeonato del mundo, que un campeonato de España. En el primer tipo de acontecimientos suele ocuparse de la seguridad las propias 


\section{La organización de grandes eventos deportivos}

Fuerzas de Seguridad del Estado, que marcan la pauta de la organización en este aspecto. En los segundos, bastan muchas veces con algún policía de seguridad privada.

En el segundo grupo de áreas, existen también dos de gran importancia y, también, muy vinculadas entre sí como son las de alojamientos y transporte por un lado, y la de instalaciones e infraestructura técnica por otro lado. Sus propias nombres ya nos indican su función y en el primer caso es aquello que no se ve en una competición o evento, pero que deja buen o mal sabor de boca, en ocasiones tan importante como la propia competición. Un buen alojamiento y una correcta recepción y traslado de los equipos, que comienza ya en el aeropuerto en las grandes ocasiones, es fundamental. Por ejemplo, la olimpiada de Atlanta será recordada por los muchos problemas de transporte que tuvo tanto como por sus posibles éxitos deportivos.

En cuanto al área técnico-deportiva es la que normalmente se encarga de los aspectos deportivos: horarios, sedes de competición, calendario, materiales, instalación, etc., y, obviamente trabaja estrechamente con la instalación.

Por último, el área médica ejerce tres tipos de funciones básicas: la atención médica a los deportistas, el control antidopaje y el establecimiento de atenciones médicas al personal de la organización y al público asistente. Dentro de la primera función se contempla también aspectos como el masaje y la fisioterapia.

\subsection{Planificación y fases de ejecución}

Desde el momento que se plantea la realización de un evento hasta su realización existen 5 fases que son las siguientes:
A.-FASE PRELIMINAR
B.-FASE DE PRESENTACIÓN
C.-FASE DE CONCESIÓN
D.-FASE DE EJECUCIÓN DE PROGRAMAS
E.-FASE DE REALIZACIÓN

De las dos primeras fases ya hemos hablado en el apartado 2.1, por lo que nos centraremos ahora en las fase C, D y E.

La primera cuestión inmediatamente después de concedido un evento será la formación del Comité Organizador, una parte importante del cual saldrá del Comité de la campaña de promoción. Es una cuestión lógica, pero a veces no ocurre así, ya que entre la concesión y la puesta en marcha de la organización suele existir una cierta relajación, 
quizás derivada de la intensidad de la campaña previa, dependiendo de qué evento se trate. Ahí se puede perder un tiempo precioso que luego debe ser recuperado de forma precipitada.

Una vez formado el Comité Organizador, la primera misión del mismo será la elaboración de los presupuestos y los programas a desarrollar en todas la áreas. Planes y programas abiertos, que tendrán que ir readaptándose a medida que la organización va cumpliendo los plazos trazados en los programas.

En esta tercera fase y al principio de la cuarta, lo más importante será la distribución de competencias entre las distintas áreas y la captación de recursos, para lo que los acuerdos comerciales (derechos de retransmisión, patrocinadores, cesión de licencias de uso de los emblemas de la competición, etc.) serán fundamentales, ya que inicialmente es posible que sean los únicos recursos con los que cuente el Comité para empezar a funcionar, puesto que las subvenciones y los ingresos por taquillas suelen venir más tarde. De hecho, estos últimos no vienen hasta el mismo año del Campeonato, como ha pasado en los dos últimos grandes acontecimientos celebrados, con el mundial de Sevilla 99 - las entradas se pusieron a la venta a finales de 1998y con la Olimpiada de Sidney, donde según sus previsiones esperaban recaudar 32.000 millones de pesetas a finales de 1999, a menos de un año de su celebración.

Al tiempo que el Comité comienza a andar y va distribuyendo las funciones aumentará de tamaño y se iniciará la contratación de personal o la captación de personal voluntario, dependiendo del evento. Todos ellos deberán contar con los dos tipos de recursos humanos, sobretodo con el voluntariado, sin los cuales no podrían celebrarse la mayoría de los grandes eventos internacionales.

Por último, la diferencia entre la cuarta y la quinta fase, ambas con tareas ejecutivas, es que en la primera de ellas se van poniendo en marcha los distintos servicios y la infraestructura necesaria para la posterior realización del evento, mientras en la segunda se realiza el evento en sí y las tareas organizativas se encaminan a cumplir los planes establecidos, controlando las contingencias que se vayan produciendo.

En ese sentido, la fase clave de toda la organización es la cuarta, pues en ella se establecerán las bases de lo que vaya a ocurrir después. $\mathrm{Si}$ el evento sale bien es porque ha sido planificado correctamente y los servicios de infraestructura reunían buenas condiciones. Si el evento va saliendo mal, las correcciones finales son difíciles porque no queda tiempo ni alternativas para modificar la fase de planificación, que es anterior. 


\section{La organización de grandes eventos deportivos}

Por ejemplo, los errores en la planificación de la infraestructura hotelera o en las necesidades de instalaciones serán prácticamente insalvables y dejarán patente los fallos organizativos. Un hotel o una instalación deportiva necesita tiempo para su construcción que ya no se tendría. Sí son salvables, en cambio, errores en el transporte (aumentar autobuses o coches, por ejemplo, se que puede arreglar) o en el material fungible o el personal necesario.

\subsection{La financiación de los grandes acontecimientos}

Los grandes acontecimientos deportivos son caros, por lo que es necesaria plantearse antes de solicitarlos los recursos con los que se va a contar porque la mayoría de ellos son deficitarios a nivel organizativo y acaban siendo cubiertos por las instituciones públicas, aunque bien es cierto que la publicidad y la estancia en la Ciudad-sede de miles de personas justifican, a veces sobradamente, esa aportación. Un ejemplo muy reciente ha sido el del Mundial de atletismo Sevilla 99: mientras que el Comité Organizador asumió un presupuesto de 3.800 millones de pesetas, con un posible déficit por inversiones de alrededor de 500 millones de pesetas, la repercusión económica alcanzó los 38.000 millones, entre inversiones y gastos de los participantes y visitantes o espectadores. Ello sin contar la repercusión publicitaria, de la que un estudio de la empresa Bassat-Ogilvi \& Mater situaba en 2.300 millones solo de la Ceremonia de Inauguración y en España. No es sería una barbaridad, ni mucho menos, situar los efectos publicitarios del Campeonato en todo el Mundo por encima de los 100.000 millones de pesetas.

Las fuentes de financiación de un acontecimiento deportivo se han diversificado en los últimos años y podemos verlos con carácter general en la el Cuadro 3.

CuAdro 3. Fuentes de financiación de un evento deportivo

- SUBVENCIONES PÚBLICAS

- DERECHOS DE RETRANSMISIÓN

- PUBLICIDAD Y PATROCINIO

- ESPECTADORES

- DERECHOS DE IMAGEN

- PUBLICACIONES 
De estas fuentes de financiación la más importante de los últimos años es la de los derechos de retransmisión televisiva, al menos en deportes como el Fútbol, Atletismo, Baloncesto, Tenis, Ciclismo y algún otro deporte. En grandes acontecimientos como las Olimpiadas estos derechos vienen a representar el 50\% de los gastos de organización, lo que es un aval para la organización que detenta estos derechos, hoy en día en manos de las Federaciones internacionales o del CIO.

Así, la retransmisión del Mundial de Fútbol de Francia produjo unos ingresos de 23.261 millones de pesetas, el de Estados Unidos de 11.124, y el de Italia en 1990 de 9.808.

En atletismo, la UER dió 16.800 millones de pesetas netos a la IAAF por un período de 6 años: 2.800 millones anuales, que en el período anterior el contrato estaba en 10.920 por 4 años: 2.730 anuales.

Y hasta un deporte que se resistía a la comercialización y profesionalización como el Rugby se acaba de rendir a este nuevo concepto del deporte y a la realidad social que nos circunda. El reciente cambio en su normativa amateur aprobada por la Federación Internacional el 27 de Agosto de 1995 ha propiciado que los derechos de retransmisión de la liga mundial se eleven a un total de 67.000 millones según el contrato que se firmo en 1998 con la Tv de Murdoch por un período de 10 años.

En el gráfico 1 podemos ver la evolución de los ingresos por retransmisiones de los Juegos Olímpicos cuyos derechos ya están vendidos hasta el año 2008 cuando aún no se sabe cual será su sede.

Estos ingresos generan las partidas más altas de los eventos deportivos, y se han multiplicado de forma impresionante desde que en 1948 el Comité Organizador de los Juegos de Londres recibiera 1500 libras por los derechos de retransmisión (Carragio, 1996, pág.139)., algo anecdótico. En Melbourne el ingreso ya fue de 51 millones de libras, lo que representó el 2,5\% de los ingresos. De estas cifras se ha pasado a los 125.515 millones de Atlanta (Diario As. 24 de julio de 1998), mientras en Barcelona 92 fueron 88.443, en Seúl 88 de 56.029 y en Los Angeles 84 de 39.894 .

Ello, a su vez, está generando ingresos para las Federaciones Internacionales, cuya distribución en Atlanta podemos ver en el Cuadro 4.

La publicidad y al patrocinio es otro elemento determinante del alcance de un acontecimiento y va parejo al nivel de audiencia del 


\section{La organización de grandes eventos deportivos}

mismo, en una relación directamente proporcional y que merece un tratamiento específico que dejamos para otra ocasión.

GRÁfICO 1. Evolución de los derechos de retransmisión de los Juegos Olímpicos

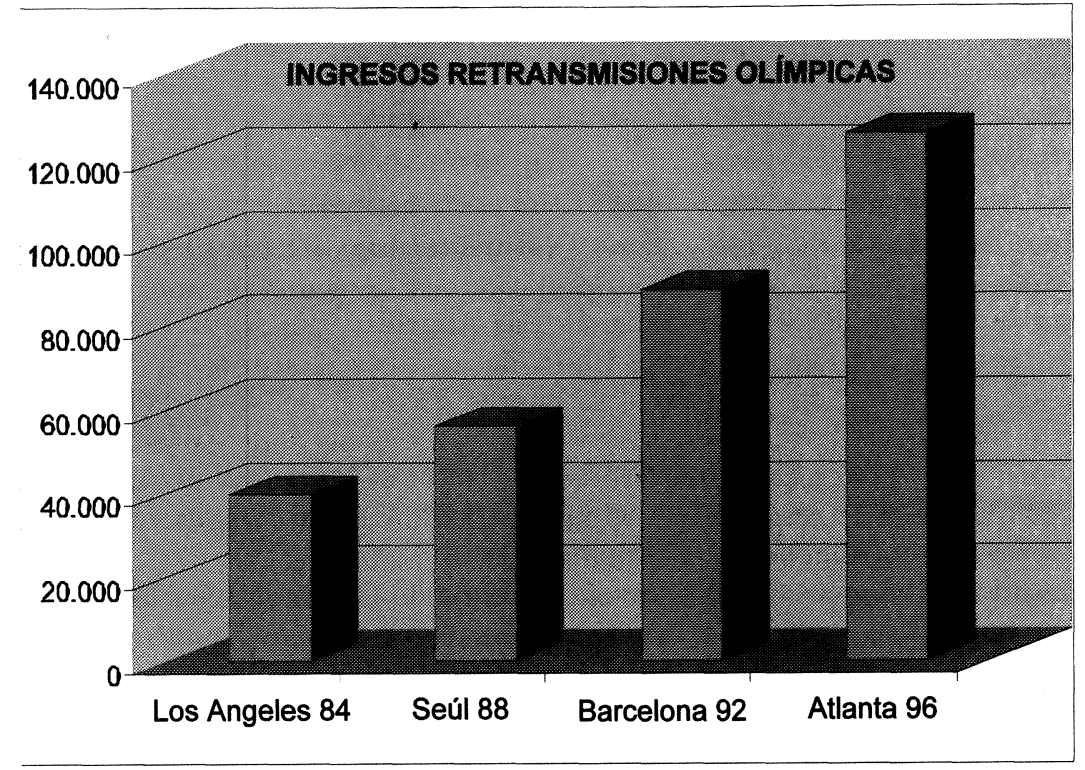

CUADRo 4. Distribución de ingresos del CIO entre la federaciones olímpicas por los derechos de Atlanta

\begin{tabular}{|l|l|l|}
\hline \multicolumn{1}{|c|}{ FEDERACIÓN } & \multicolumn{1}{|c|}{$\begin{array}{c}\text { CANTIDAD POR } \\
\text { PUBLICIDADY } \\
\text { COMERCIALIZACIÓN }\end{array}$} & \multicolumn{1}{c|}{$\begin{array}{c}\text { POR DERECHOS } \\
\text { DE TV }\end{array}$} \\
\hline ATLETISMO & 780 MILLONES & 252 MILLONES \\
\hline $\begin{array}{l}\text { FÚTBOL, VOLEIBOL, } \\
\text { BALONCESTO, NATACIÓN } \\
\text { Y GIMNASIA }\end{array}$ & 300 MILLONES CADA UNA & 252 MILLONES CADA UNA \\
\hline $\begin{array}{l}\text { TENIS, HOCKEY, REMO, } \\
\text { BALONMANO, CICLISMO } \\
\text { Y EQUITACIÓN. }\end{array}$ & 120 MILLONES CADA UNA & 252 MILLONES CADA UNA \\
\hline 14 DEPORTES RESTANTES & 60 MILLONES CADA UNA & 252 MILLONES CADA UNA \\
\hline
\end{tabular}


Por otra parte, el merchandising y los derechos de imagen son conceptos nuevos que va introduciéndose cada vez más en el deporte de forma más profesionalizada en vista, sobretodo, del éxito del mismo en países como Estados Unidos o Gran Bretaña (Sleight, 1992; Mullin et alt.,1995), donde un equipo como el Manchester ingresa del orden de 4.000 millones de pesetas por este concepto.

\section{Notas}

1 Juan A. Samaranch fue elegido Presidente del CIO en 1980 en el Congreso celebrado en Moscu. Sus reformas no pudieron ser inmediatas sino que empezaron a plasmarse a partir de la Olimpiada de Los Angeles.

2 Matanza de estudiantes en la Plaza de las Tres Culturas en México y manifestación en el podium de los ganadores negros de las pruebas de 200 y 400 metros en defensa del Black Power; secuestro y muerte de deportistas israelíes y de propio comando palestino que les secuestró en Munich; boicot de los países africanos a Montreal.

3 Ello a pesar de la cercanía a la olimpiada de Barcelona, 12 años, misma diferencia que hubo entre Los Angeles 84 y Atlanta 96.

4 Desde la olimpiada de 1952 nunca ha repetido Continente en las nominaciones olímpicas. Helsinki en 1952 fue la última al suceder a la de Londres de 1948.

5 La Federación Internacional de Atletismo finalmente ha entendido el tema y de cara a los próximos años existe intención de conceder de golpe los campeonatos del año 2003 y 2005, al menos.

\section{Bibliografía}

AÑó, V. (1991): Organización del Campeonato del Mundo de atletismo en pista cubierta. En Jornadas sobre Organización grandes eventos internacionales. Universidad M. Pelayo. Barcelona.

AÑó, V. (1993): La gestión de las Federaciones Deportivas. Ed. AEISAD. PAMPLONA.

AÑó, V. (1995): Políticas deportivas y financiación. Congreso L' esport. Valencia.

BRoHM, JM. (1976): Sociologie politique du sport. Ed.JP.Delarge. Paris.

Carragio, M. (1996). Patrocinio Deportivo. Ed. Ariel Comunicación. Barcelona.

Elias, N., y Dunnig, E. (1992): Deporte y ocio en el proceso de civilización. Ed. FCE. México.

Kotler, P., Cámara, D., y Grande, I. (1995. $8^{\mathrm{a}} .2^{\mathrm{a}}$ española): Dirección de Marketing. Ed. Prentice Hall. Madrid.

Mandell, R. (1986): Historia Cultural del Deporte. Ed. Bellaterra. Barcelona.

MintZBerg, H. (1983): La naturaleza del trabajo directivo. Ed.Ariel. Barcelona.

Muluin, B., Hardy, S., y Sutton, W. (1995): Marketing Deportivo. Ed. Paidotribo. Barcelona.

Peiró, JM. (1986): Psicología de la Organización. Ed. U. N. Educación a Distancia. Madrid. 


\section{La organización de grandes eventos deportivos}

RAPP, S., y Collins, T. (1987): Maximarketing. MacGraw \& Hill. N.York.

Santesmases, M. (1996): Términos de Marketing. Ed. Piramide. Madrid.

Simson, V. y Jennings, A. (1992): The lords of de rings. Ed. Simon \& Schuster. London.

SLeight, S. (1992): Patrocinadores. Ed. Macgraw \& Hill. Madrid. 